OPEN ACCESS

Edited by:

Daniel Gyamfi Amoako,

National Institute for Communicable

Diseases (NICD), South Africa

Reviewed by:

Gisela Unis,

Hospital Sanatorium Partenon

(HSP), Brazil

Muhammad Furqan Akhtar.

Riphah International University

(Lahore), Pakistan

Ayo Ajayi,

Federal University Oye Ekiti, Nigeria Htin Lin Aung,

University of Otago, New Zealand

${ }^{*}$ Correspondence: Ngula Monde

nkabelenga@yahoo.co.uk

Specialty section: This article was submitted to Antimicrobial Resistance, a section of the journal Frontiers in Tropical Diseases

Received: 01 July 2021 Accepted: 06 October 2021 Published: 03 November 2021

Citation:

Monde N, Zulu M, Tembo M, Handema R, Munyeme $M$ and Malama S (2021) Drug Resistant Tuberculosis in the Northern Region of Zambia: A Retrospective Study.

Front. Trop. Dis. 2:735028. doi: 10.3389/fitd.2021.735028

\section{Drug Resistant Tuberculosis in the Northern Region of Zambia: A Retrospective Study}

\author{
Ngula Monde ${ }^{1,2^{*}}$, Mildred Zulu ${ }^{2,3}$, Mathias Tembo ${ }^{1}$, Ray Handema ${ }^{1}$, Musso Munyeme $^{2}$ \\ and Sydney Malama ${ }^{4}$ \\ ${ }^{1}$ Department of Biomedical Sciences, Tropical Diseases Research Center, Ndola, Zambia, ${ }^{2}$ Department of Disease Control, \\ School of Veterinary Medicine, University of Zambia, Lusaka, Zambia, ${ }^{3}$ Department of Pathology and Microbiology, School of \\ Medicine, University of Zambia, Lusaka, Zambia, ${ }^{4}$ Department of Biological Sciences, School of Natural Science University of \\ Zambia, Lusaka, Zambia
}

Background: Zambia like many countries in sub-Saharan Africa is affected with drug resistant tuberculosis. However, the drug resistant tuberculosis situation over the years has not been described in various regions of the country. Consequently, this study aims to determine the drug resistant tuberculosis burden in northern regions of Zambia over a four-year period based on data generated from a Regional Tuberculosis Reference Laboratory.

Method: Two hundred and thirty two (232) Tuberculosis Drug Susceptibility Testing results over a four-year period (2016-2019) were reviewed. Data was collected from tuberculosis registers and patient request forms and entered into a pre-tested standardized checklist and later entered in Excel Computer software. Double blinded checking was done by two independent data clerks to minimize duplication of cases. Cleaned data was then imported in R programme for analysis. Bivariant and descriptive statistics were performed and reported.

Results: Of 232 Drug Susceptibility Testing results, 90.9\% were drug resistant TB while $9 \%$ were drug susceptible. Fifty three percent (53\%) of these were multi-drug resistant Tuberculosis and $32 \%$ were confirmed as Rifampicin Mono-resistance. Only $1.7 \%$ of the Multi-drug resistant Tuberculosis patients were Pre-extensively drug-resistant Tuberculosis. Copperbelt province had the largest proportion $(46.0 \%)$ of multi-drug resistant tuberculosis patients followed by Luapula (8.1\%) and North-Western (4.7\%) provinces. In new and previously treated patients, the proportion of Multi-drug resistant tuberculosis was $71.8 \%$ and $28.7 \%$ respectively. History of previous anti-tuberculosis treatment and treatment failure were associated with multi-drug resistance TB.

Conclusion and Recommendation: This study has shown a small increase in the proportions of drug resistant tuberculosis cases over the four years under review with high 
rates being recorded on the Copperbelt Province. Previous treatment to first line TB treatment and treatment failure were associated with development of Multi-drug resistance. We therefore recommend strengthened routine laboratory surveillance and improved case management of multi-drug resistant tuberculosis patients in the region.

Keywords: tuberculosis, drug resistance TB, multi-drug resistance TB, Zambia, Northern region

\section{BACKGROUND}

Globally, Tuberculosis (TB) is a public heath challenge causing a number of mortalities. It is caused by an infectious bacterial agent known as the Mycobacterium tuberculosis complex. Approximately 10 million individuals were infected with the disease in 2019 with 1.2 million HIV negative and 208, 000 HIV positive individuals dying from the disease (1). The highest morbidity and mortality rates of TB are mainly reported in the low and middle income countries, Zambia inclusive (2). Global, regional and national efforts are being made through End TB Strategy by WHO to lower the TB incidence and number of deaths by approximately 90\% and 95\% respectively by 2035 compared to levels in 2015 (1).

Drug resistant tuberculosis (DR TB), particularly, rifampicin resistant Tuberculosis (RR-TB), Multidrug resistant Tuberculosis (MDR-TB) and extensively drug-resistant Tuberculosis (XDR-TB) continue to be one of the leading public health problem that threatens global TB control and prevention (3). Multi drug resistant refers to TB bacteria that is resistant to at least rifampicin (RIF) and isoniazid (INH), the most two, potent anti-TB drugs whereas, Extensive drugresistant $\mathrm{TB}$ refers to $\mathrm{MDR}-\mathrm{TB}$ which is also resistance to two groups of secondline ati-TB drug (a fluoroqurinolone (FQ) and a second-line injectable drug) $(4,5)$. Rifampicin Resistance is a surrogate for MDR-TB and WHO recommends prompt detection and diagnosis of RR-TB. World Health Organization 2020 global TB report shows that $3.3 \%$ and $18 \%$ of new and previously treated $\mathrm{TB}$ cases had MDR-TB respectively. Approximately half a million people had RR-TB of which $78 \%$ developed MDR-TB and 6.2\% of MDR TB patients had XDR-TB. In 2019, about 0.5 million people were diagnosed or notified with MDR-TB/RR-TB with only one in three people being enrolled on treatment. Only $56 \%$ of those enrolled on treatment were successfully treated (1). Approximately 50\% of MDR/RR-TB cases were reported in India (27\%), China (14\%) and Russian federation (9\%) (1). Africa, particularly Sub-Saharan Africa (SSA) is among the regions affected by the emergence of drugresistance TB $(6,7)$. Data on drug resistant TB in most countries in Africa is limited $(2,8,9)$. In the recent past, only few countries had data on the burden of Drug resistant TB based on their periodic national DR TB surveys $(1,8)$. Therefore, a number of studies were conducted to estimate the burden of MDR-TB in Sub-Saharan Africa. Lukoye et al. found a pooled prevalence of MDR-TB of $1.5 \%$ (95\% CI: 1.0 - 2.3) (10), while a meta-analysis study done by Musa et al., reported a pooled prevalence of $2.1 \%$ (95\% CI: 1.7 - 2.4) among new TB cases, representing a relatively low prevalence of MDR-TB cases in SSA (7). Currently, the expansion of rapid molecular tests has resulted in a number of countries transitioning from reliance on DR TB Survey data to continuous surveillance system data resulting in improved data collection and analysis by WHO. In 2019, WHO reported an estimated percentage of MDR/RR-TB of $2.6 \%$ and $11 \%$ in new cases and previously treated patients. The percentage of RR-TB within MDR-TB was found to be $76 \%$ (1).

Zambia is among the countries in sub-Saharan Africa with a high burden of TB. It has a population of 13.1 million people and $44 \%$ of the population live in urban areas (11). In 2018, WHO estimated the total incidence of TB at 346/100 000 population per year. The TB incidence in HIV positive individuals was 205/ 100,000 population with 59\% HIV prevalence in new and relapse TB cases of all ages. Regardless of achieving a good treatment success rates for drug susceptible TB over the years, drug resistant $\mathrm{TB}$ remains a growing health problem in Zambia (12). Previous reports from national surveys conducted in 2001 and 2008 had reported a relatively low prevalence of MDR TB in Zambia $(12,13)$. In 2001, the prevalence of MDR TB based on the national DR TB survey was $1.2 \%$ and $1.8 \%$ in new and previously treated TB patients (13). Similarly, the 2008 national drug resistance survey showed an MDR TB prevalence of $0 \%$ in new cases and $6.5 \%$ in previously treated TB cases. Resistance to any drug was at $9.8 \%$ among new TB cases (12). On the other hand, a recent review of national data for a period of 11 years (2000 to 2011) showed an increase in the number of MDR TB cases diagnosed each year through routine surveillance system (14). Further, in 2018, the WHO Global TB report showed an estimated 1,500 MDR/Rifampicin resistant TB (RR-TB) patients among notified pulmonary TB patients in Zambia and the prevalence of MDR-TB/RR-TB in Zambia was about $2.8 \%$ in new patients and $18 \%$ in previously treated patients (1). Other studies on prevalence of drug resistance in Zambia include those conducted in 13 Zambian prisons and a cross sectional explorative study on prevalence of Rifampicin resistance in TB patients at livingstone Central Hospital $(15,16)$. The prevalence of MDR TB in prison inmates was 9.5\% (16) and that of rifampicin resistance in the latter study was 5.9\% (15).

Mycobacterium Tuberculosis drug resistance can be acquired or primary. Acquired DR TB occurs when TB treatment is inadequate. This can be due to deficient policies and failures in health care, wrong prescription of $\mathrm{TB}$ drugs or substandard drugs being used for treatment and patients poor treatment compliance. On the other hand, primary DR TB refers to direct transmission of drug resistant Mycobacterium tuberculosis strains from person to person (1).

Tuberculosis drug resistance is mainly caused by mutations in genes that codes for anti-TB drug target and the mutations are 
primarily insertions or deletions (indels) and Single Nucleotide Polymorphisms (17). Recent studies on drug resistance development show that isoniazid resistance happens much earlier, followed by resistance to rifampicin or ethambutol, then resistance to pyrazinamide and lastly, resistance to second- and third-line drugs (18-20). Resistance to Isonaizid $(\mathrm{INH})$ is as a result of mutations in the katG gene and the promoter region of inhA. Among the katG mutations, the S315T (accounts for 40 to $94 \%$ resistance in MDR strains) and the newly reported L101R are the most common mutations associated with high level INH resistance (21). The most prevalent mutation in inhA promoter region is the c- $15 \mathrm{t}$ mutation which accounts for about $19 \%$ of INH resistance and is associated with low level resistance to INH $(\mathrm{MIC}<1 \mu \mathrm{g} / \mathrm{ml})(22-24)$. Double mutations such as the $-8 \mathrm{~T} / \mathrm{C},-15 / \mathrm{T}(25)$, and $-17 \mathrm{C} / \mathrm{T}$ (26) in the promoter region of inhA have also been known to be associated with INHresistance. Likewise, Rifampicin resistance is caused by mutations in codons 507-533 of a gene coding for the beta subunit of the RNA polymerase known as the $r p o B$ gene. Most of the RIF-resistance (96\%) occurs within RIF-resistance determining region (RRDR), also known as the "hot-spot region" (81 bp), covering codons 507-533 of rpoB gene (27) and this region is the target for most current TB molecular-based assays. The most prevalent mutations known to be associated with RIF resistance occurs in codons 516, 526 and 531 of the RRDR (21). Mutations outside the RRDR have also been reported in rifampicin-resistant isolates (28). Drug resistance genes that infer resistance in secondline TB drugs have also been identified and these consists of the gyrA and gyrB (flouroquinolones), rrs (Streptomycin, Amikacin, Kanamycin, Capreomycin), inhS, othA and ethR (Ethionamide), alr and ddl for (D-cycloserine) and eis (Kanamycin) $(9,29)$.

Laboratory investigations are critical in the clinical assessment of patients and guide clinicians in the management of patients. One of the primary elements in the strategy to control TB is effective case management through accurate and early diagnosis and treatment of TB patients $(9,30)$. Phenotypic drug susceptibility testing (DST) is the gold standard for the diagnosis of Drug Resistant tuberculosis $(31,32)$. The diagnosis of DR-TB in Zambia is currently being done using GeneXpert MTB/RIF (Xpert MTB/RIF), Line Probe Assay (LPA) (first- and secondline) and phenotypic drug-susceptibility testing.

In 2011, WHO recommended Xpert MTB/RIF testing as the preferred rapid molecular diagnostic test for rifampicin resistance (RR) for all TB presumptive patients. In Zambia, the National tuberculosis and Leprosy control Programme endorsed the WHO recommendations in 2012 and recommended Xpert MTB/RIF as the firstline diagnostic tool for high-risk presumptive TB patients. In 2017, GeneXpert MTB/RIF was adopted as the firstline diagnostic test for all presumptive TB patients regardless of risk factors, to improve case detection for susceptible and drug resistant TB. In order to improve access of patients to this diagnostic tool, the Xpert MTB/RIF machines are placed at TB diagnostic centers countrywide as close as possible to the patients in the communities (33). However, despite this, there is low uptake of this diagnostic tool. To further improve
DR-TB diagnosis, Line Probe Assays (LPA) such as GenoTypeMTBDRplus and GenoTypeMTBDRsl assay (Hain Life Science GmbH, Nehren, Germany) are available for the detection of MDR-TB and early detection of resistance to key second line DR-TB drugs (Fluoroquinolones and injectables). However, this technology has only been implemented at the National Reference Laboratory (Chest Diseases Laboratory in Lusaka) and two regional TB reference laboratories (University Teaching Hospital in Lusaka and Tropical Diseases Research Centre in Ndola) and not available at point of care. The three facilities are $\mathrm{TB}$ culture laboratories and have the capacity to perform first and second line phenotypic and Genotypic Drug Susceptibility Testing. Samples for all patients diagnosed with rifampicin resistance detected on GeneXpert MTB/RIF from the various TB diagnostic facilities countrywide are referred to the aforementioned culture facilities for Firstline and Secondline LPA, culture, and phenotypic DST. The patients are then commenced on the shorter DR-TB treatment regimen (if eligible) while waiting for the results. The National Tuberculosis and Leprosy Programme has adopted the WHO recommendations, shorter regimen ( 9 to 12 months) in patients without resistance or intolerance to key second-line drugs, including the fluoroquinolones (FQ) and/or second-line injectables (SLIs). For patients that are not eligible for the shorter regimen, an individualized treatment regimen is designed containing new drugs such as bedaquiline (Bdq) and delamanid (Dlm) and repurposed drugs like linezolid (Lzd) and clofazimine (Cfz) (34).

Zambia is a landlocked country. It is also a copper producing country mainly from the Copperbelt and North-Western provinces. As such there is frequent movement of individuals and goods into the neighboring countries which own ports (South Africa, Tanzania, Mozambique and Namibia) and beyond. During these cross border movements drug resistant tuberculosis can easily be spread. Therefore, it is important to know the burden of drug-resistant TB in the various regions of the country, particularly the Northern Region. Additionally, there is limited data in the literature on the burden of drug resistant $\mathrm{TB}$ in different regions of the country. It is against this background that this study aims at determining the burden of drug resistant tuberculosis in the Northern Region of Zambia from 2016 to 2019 based on analyzed TB samples with DST results at the Regional Reference laboratory. The findings of the study are envisioned to help in strengthening laboratory drug resistant TB surveillance, improving management of patients with Drug resistant TB and control of the disease.

\section{METHODOLOGY}

\section{Study Design}

This was a retrospective study where all TB Drug Susceptibility Testing (DST) results from processed TB samples at the Regional TB reference culture Laboratory from January 2016 to December 2019 were considered. The TB samples were referred to the regional reference laboratory from the Northern region of 
Zambia and each sample represent a different patient. The period for the study was selected because diagnosis of DR TB using Line probe assay at the Reference laboratory was implemented in 2016. The regional TB Reference laboratory is the only laboratory in the Northern Region of Zambia that provides culture and DST services. This Region comprises three provinces namely; Copperbelt, North Western and Luapula (Figure 1). All clinical TB samples from Rifampicin Resistant, suspected MDR TB and pre-XDR cases from the various TB diagnostic health facilities in the region are referred to this laboratory for MDR TB, Pre-XDR TB and XDR TB diagnosis through culture and DST.

\section{Inclusion Criteria}

All complete TB DST results by Line Probe Assay were included in this study (Figure 2).

\section{Exclusion Criteria}

All contaminated, Non-tuberculous Mycobacteria (NTM), missing culture results and incomplete demographic data were excluded from this study.

\section{Data Collection}

Data on socio-demographic variables, DST results (based on Line Probe Assay) were extracted from TB laboratory register and request forms using a predesigned checklist. Patients that were diagnosed with DR-TB in the previous years were removed in subsequent years to minimize duplication of data entry. The data was also verified for consistency and completeness.

\section{Data Management}

Data was collected from TB registers and patient request forms and entered into a pre-tested standardized checklist, which was later entered in Excel Computer software. Double blinded checking was done by two independent data clerks to minimize duplication of cases. Cleaned data was then exported to R programme (35), for analysis. Descriptive statistics were performed and reported as frequencies and graphs. Chi square test was employed to determine the association between various factors with MDR TB. A p-value of 0.05 was considered statistically significant at $95 \%$ confidence Interval.

\section{RESULTS}

\section{Characteristics of the Drug-Resistant TB Patients}

Out of 232 samples with DSTs results, 211 (90.9\%) were drug resistant TB while 21 (9\%) were drug susceptible. The majority $170(73.3 \%)$ of the patients were males, and $147(63.3 \%)$ were in the age group of 30 to 49 years with the mean age of 35.59 $(\mathrm{SD}=10.9)$ years. One hundred and four patients $(44.8 \%)$ were co-infected with HIV (Table 1). The majority of the patients came from Copperbelt province $188(81 \%)$ followed by Luapula $24(10.3 \%)$ and then North Western $20(8.6 \%)$ provinces. Of the DR TB patients, $29.7 \%$ were new DR patients while $61.6 \%$ were re-treatment cases (Table $\mathbf{1}$ ).

\section{Proportions of Drug-Resistant TB Cases}

The proportions of Drug Resistant TB cases diagnosed at the TB reference lab from 2016 to 2019 are as shown in Table 2.

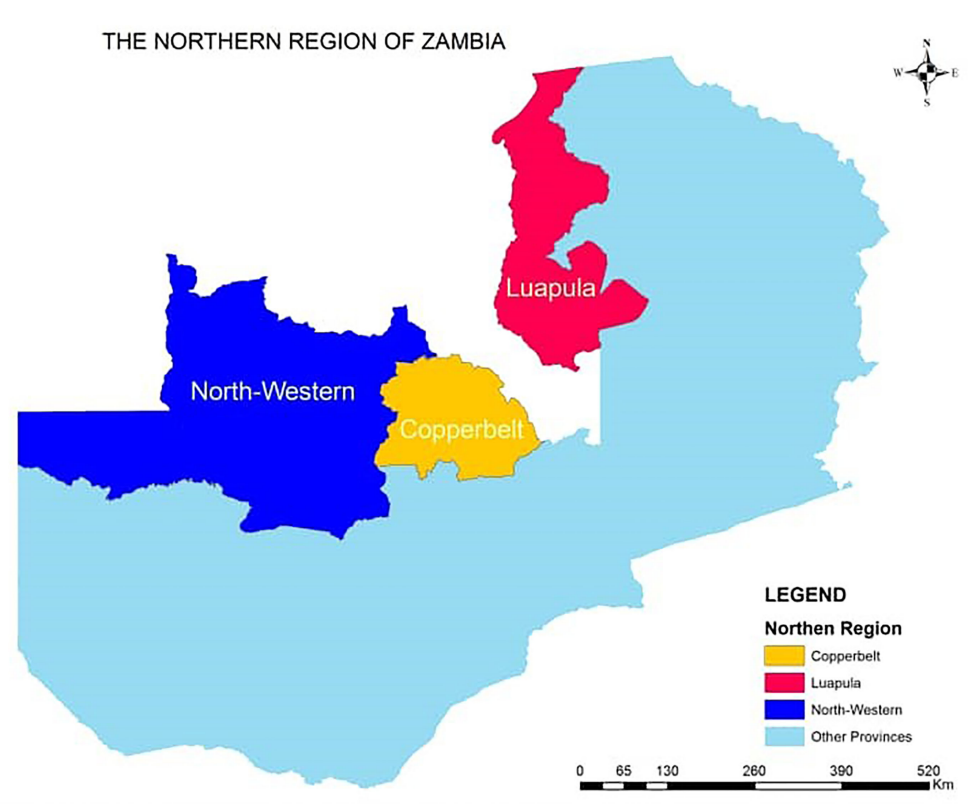

FIGURE 1 | Map of Zambia showing the provinces in the Northern Region supervised by TB regional reference laboratory, TDRC. 


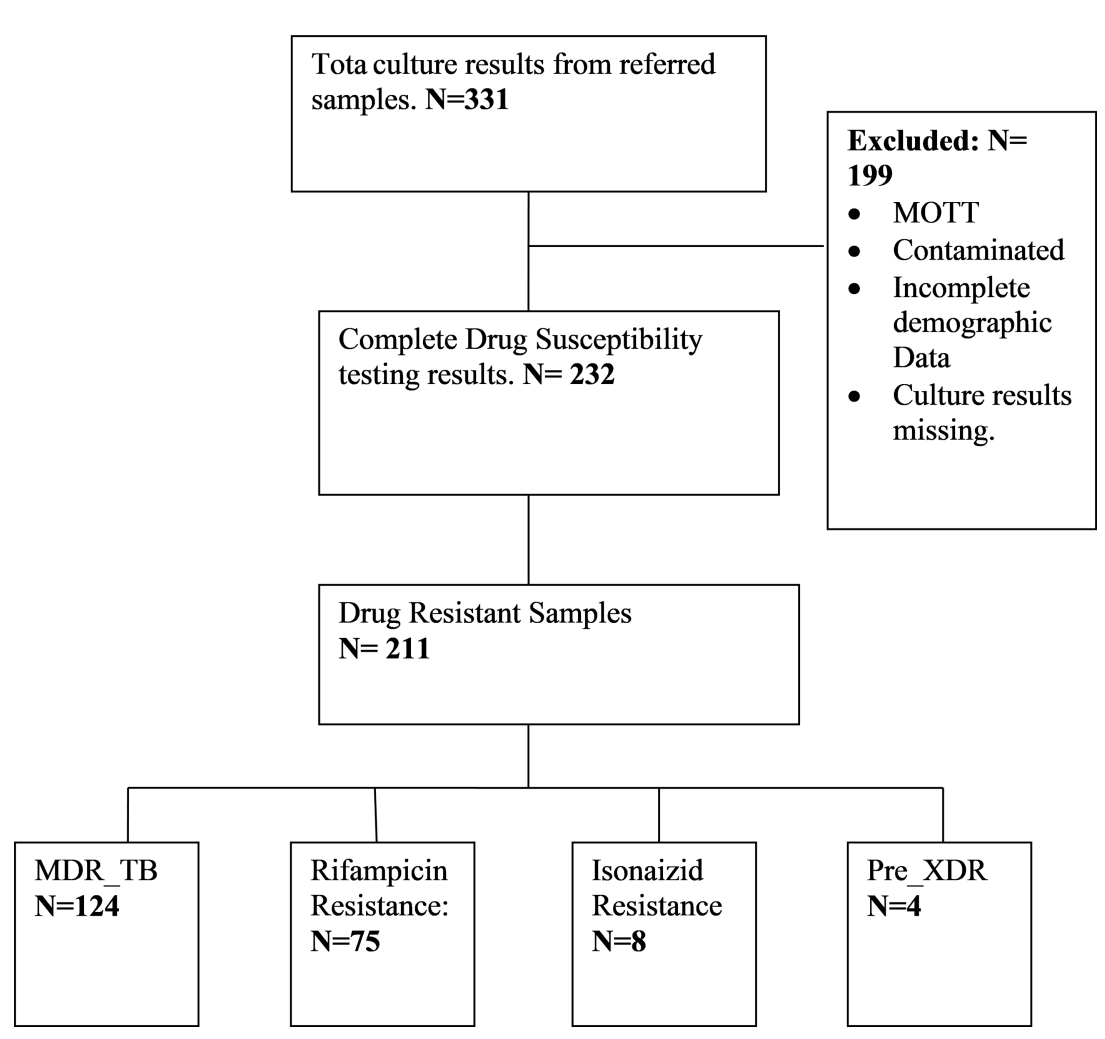

FIGURE 2 | Flow chart showing the selection criteria of data included in the review.

TABLE 1 | Social demographic characteristics and clinical information of DR TB patients $\mathrm{N}=232$.

\begin{tabular}{lcc}
\hline Variable & N & $\%$ \\
\hline Gender: & 170 & \\
$\quad$ Male & 62 & 73.3 \\
Female & & 26.7 \\
Age Category: & 63 & \\
$\quad<29$ years & 147 & 27.1 \\
$30-49$ years & 22 & 63.3 \\
$>50$ years & & 9.5 \\
HIV Status: & 128 & 55.2 \\
Negative & 104 & 44.8 \\
Positive & & \\
Previous history of TB treatment: & 69 & 29.7 \\
New & 143 & 61.6 \\
Re-treatment & 9 & 3.9 \\
MDR Contact & 11 & 4.9 \\
Uncertain & & \\
Province: & 188 & 81.0 \\
Copperbelt & 24 & 10.3 \\
Luapula & 20 & 8.6 \\
North-Western & & \\
\hline
\end{tabular}

MDR, Multi-drug Resistance.

The majority $124(53.4 \%)$ of the Drug Resistant results were MDR TB, 75 (32.3\%) were Rifampicin mono-resistant, 8 (3.4\%) were Isonaizid mono resistant and $4(1.7 \%)$ were Pre XDR-TB. Of the pre-XDR TB, 3 were resistant to second line injectable drugs and one case was resistant to Fluoroquinolones only.
TABLE 2 | Proportion of Drug Resistant TB cases among 232 Drug Susceptibility Testing by Line Probe assay from 2016 to 2019.

\begin{tabular}{lcc}
\hline DST & Number & Proportion \\
\hline Susceptible & 21 & $9.1 \%$ \\
Isonaizid Mono-resistance & 8 & $3.4 \%$ \\
Rifampicin Mono-resistance & 75 & $32.3 \%$ \\
Multi-drug Resistance & 124 & $53.4 \%$ \\
Pre-XDR TB* & 4 & $1.7 \%$ \\
\hline${ }^{*}$ Pre-XDR TB, Pre-extensively Drug Resistant & Tuberculosis; DST, Drug Susceptibility \\
Testing. &
\end{tabular}

\section{Drug Resistant TB Cases by Year and Province}

Figure 3 shows a gradual increase in Rifampicin resistant TB from 2016 to 2019. Review of drug resistance data by province showed that most $(46.6 \%)$ of the patients with MDR-TB were from the Copperbelt, followed by Luapula (8.1\%) and then North Western (4.7\%) provinces (Figure 4).

\section{Factors Associated With Multi-Drug Resistant TB}

Table 3 summaries the factors associated with MDR TB among drug resistant TB cases. There was a significant difference between having MDR TB and having been previously treated OR $2.38(1.33$ - 4.24) Pvalue 0.003 . The odds of having MDR TB when one has been previously treated for TB was 2.38 times (138\% higher than) the 


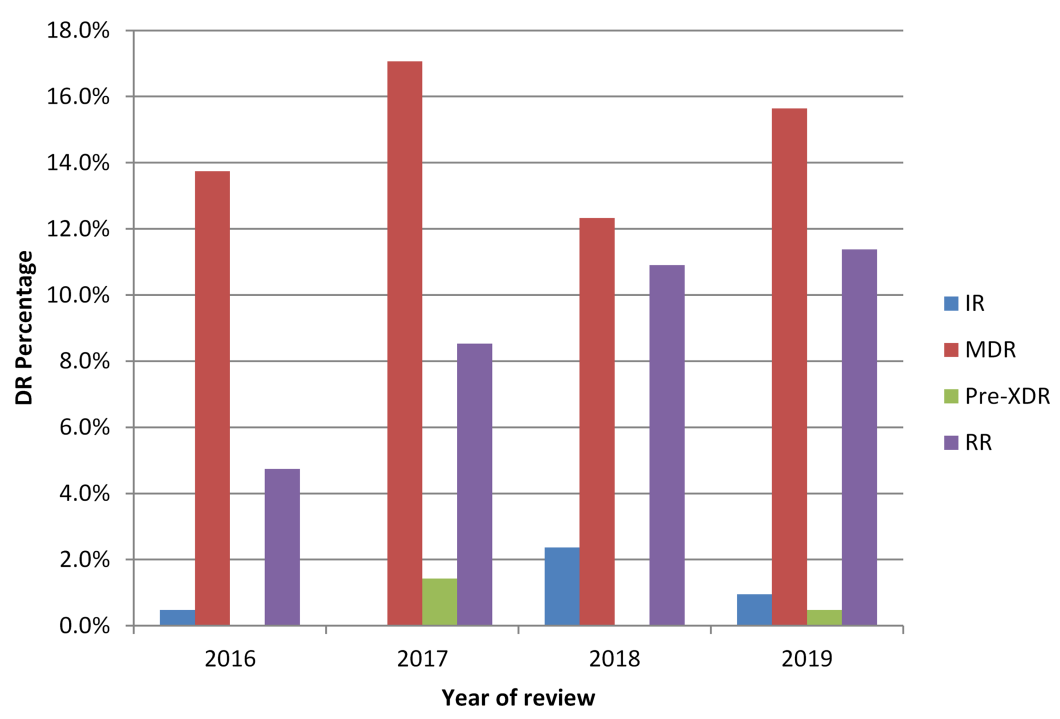

FIGURE 3 | Drug Resistant cases in the Northern Region of Zambia over a period of four years, N = 211. DR, Drug Resistance; IR, Isonaizid Resitance TB; MDR, Multi Drug Resistance TB; Pre-XDR, Pre-XDR, Pre-Extensive Drug Resistant TB; RR, Rifampicin Resistance TB.

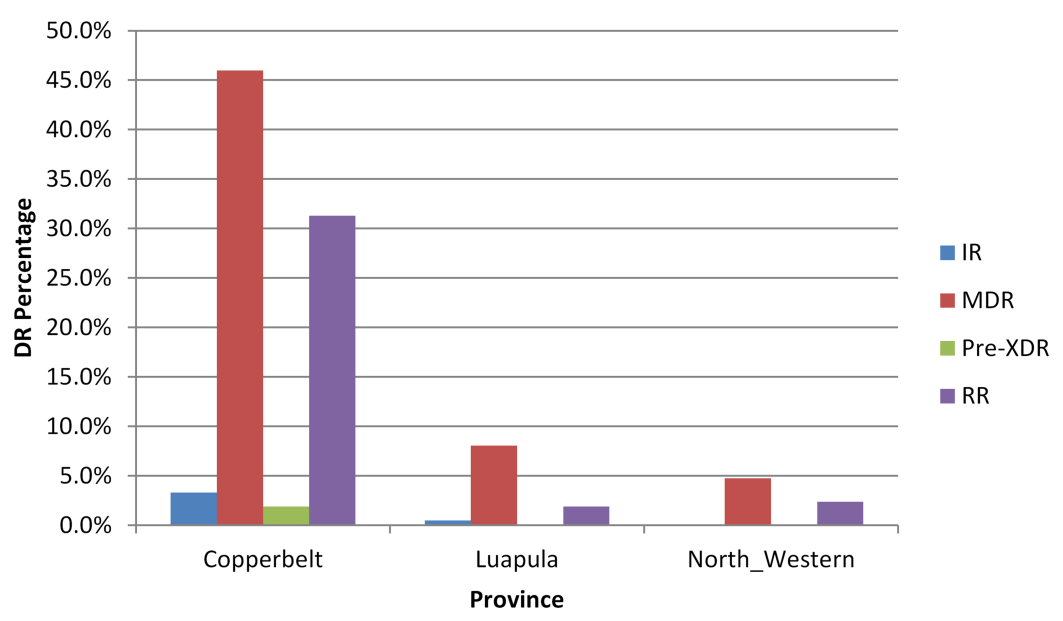

FIGURE 4 | Drug Resistant TB Cases in three provinces of the Northern Region of Zambia, N = 211. DR, Drug Resistance; IR, Isonaizid Resitance TB; MDR, Multi Drug Resistance TB; Pre-XDR, Pre-XDR, Pre-Extensive Drug Resistant TB; RR, Rifampicin Resistance TB.

odds of having MDR TB when one has not received any TB treatment. There was also a significant difference between having MDR TB and treatment failure to firstline anti-TB drug OR 2.41 (1.35 - 4.30), P-value 0.002. The odds of having MDR TB when one has failed treatment to firstline anti-TB drugs was 2.41 times (141\% higher than), the odds of having MDR TB when one has not had any treatment failure.

\section{DISCUSSION}

This study aimed at determining the burden of drug resistant tuberculosis in the Northern Region of Zambia based on TB DST results from samples analyzed at the Regional Reference laboratory. Our study has shown that progress being made to control the global tuberculosis (TB) epidemic is currently complicated by the emergence of strains of Mycobacterium tuberculosis Complex which are resistant to one or more anti-tuberculosis drugs. In Zambia there is insufficient published data regarding the burden of drug resistant $\mathrm{TB}$ in the various regions of the country as the last MDR national survey was conducted in 2008 (12). Overall, the proportion of MDR TB in this current study was 53\%. Although, other studies have reported a much lower prevalence of MDR-TB (36-38), our study has shown a higher proportion of MDR TB cases among the culture positive samples analyzed for DST at the regional 
TABLE 3 | Factors associated with MDR TB (N=211).

\begin{tabular}{|c|c|c|c|c|}
\hline Variable & MDR_TB $(n=128)$ & Non MDR TB $(n=83)$ & OR $(95 \% \mathrm{Cl})$ & P-value \\
\hline \multicolumn{5}{|l|}{ Gender: } \\
\hline Male & $97(75.8)$ & $59(71.1)$ & $1.28(0.68-2.37)$ & 0.452 \\
\hline Female & $31(24.2)$ & $24(28.9)$ & 1 & - \\
\hline \multicolumn{5}{|c|}{ Age Category: } \\
\hline$\leq 40$ years & $85(66.4)$ & 60 (72.3) & 1 & - \\
\hline$>40$ years & 43 (33.6) & $23(27.7)$ & $1.32(0.72-2.42)$ & 0.373 \\
\hline \multicolumn{5}{|l|}{ HIV Status: } \\
\hline Negative & $74(57.8)$ & $43(51.8)$ & 1 & - \\
\hline Positive & $54(42.2)$ & $40(48.2)$ & $0.78(0.45-1.37)$ & 0.396 \\
\hline \multicolumn{5}{|c|}{ Previous Treatment: } \\
\hline Yes & $92(71.8)$ & $43(51.8)$ & $2.37(1.33-4.24)$ & 0.003 \\
\hline No & $36(28.1)$ & $40(48.2)$ & 1 & - \\
\hline \multicolumn{5}{|l|}{ TB Relapse } \\
\hline Yes & $67(52.3)$ & $26(31.3)$ & $2.41(1.35-4.29)$ & 0.002 \\
\hline No & $61(47.7)$ & $57(68.8)$ & 1 & - \\
\hline \multicolumn{5}{|c|}{ Copperbelt Province: } \\
\hline Yes & 101 (78.9) & $73(88.0)$ & $0.512(0.23-1.12)$ & 0.093 \\
\hline No & $27(21.1)$ & $10(12.0)$ & 1 & - \\
\hline
\end{tabular}

MDR-TB, Multi-drug resistant tuberculosis; 95\% Cl, 95\% Confidence Interval.

The bold numbers means they are Statistically Significant.

reference laboratory. Similarly there has been a small and gradual increase in the cases of Rifampicin Resistant TB from 2016 to 2019. This increase in the cases of drug resistance TB could be attributed to either improved diagnosis of Drug resistant TB as evidenced by the increased rollout of the rapid molecular diagnostic tool (GeneXpert) or poor treatment outcomes resulting in increased transmission of DR TB in the community.

The proportion of MDR-TB among new and previously treated patients was $71.8 \%$ and $28.1 \%$ respectively. History of previous anti-tuberculosis treatment was found to strongly associated with MDR TB $(\mathrm{p}=0.003)$. Those who were previously treated for TB had higher odds of developing MDRTB. The finding is consistent with other studies conducted in Europe, Nigeria and Malaysia (39-41). The MDR TB patients in our study have had treatment to first line anti-TB treatment at one point in time. Similarly, there was an association between patients who had treatment failure and having MDR-TB. Those who had treatment failure to first line TB drugs had higher odds of developing MDR TB. This finding may be linked to poor patient treatment compliance, irregular drug supply and drug malabsorption leading to the bacteria developing resistance.

Further, $1.7 \%$ of the MDR-TB patients had Pre-extensively Drug Resistant TB (Pre-XDR TB). Pre-XDR-TB is MDR-TB strain that is resistant to either Fluoroquinolones (FQ) or second line injectable drugs (Capreomycin, Kanamycin or Amikacin) but not both. Equally this finding is comparable to a finding in a similar study by Shibabaw and colleagues conducted in Ethiopia where $6.3 \%$ of MDR TB isolates were reported to be resistant to at least one-second line TB drugs. Pre-XDR-TB and XDR-TB isolates accounted for $5.7 \%$ and $0.6 \%$ respectively (42). On the contrary, other studies conducted on the same phenomenon in other African countries like Nigeria and South Africa have actually reported much higher rates of pre-XDR TB of $16.7 \%$, and $17 \%$ respectively $(43,44)$. The higher rate of pre-XDR cases in these countries could be due to better laboratory setup and resources for the isolation of drug resistant strains. Identification of pre-XDR TB patients is cardinal as it assists clinicians to monitor the patients closely and prevent the progression to $\mathrm{XDR}-\mathrm{TB}$ which is more difficult to treat and has poor treatment outcome.

Additionally, 3.4\% of the cases diagnosed were Isonaizid monoResistant (IR). Isonaizid mono-resistance is much more common than Rifampicin mono-Resistant TB and is associated with treatment failure, relapse and progression to multidrug-resistant TB. This study is also in tandem with findings by Dean and colleagues on aggregated drug resistance data reported to WHO from both routine continuous surveillance and nationally representative periodic surveys of $\mathrm{TB}$ patients for the period 2003-2017. Their analysis showed that on average, 7.4\% of new cases and $11.4 \%$ of previously treated patients had IR-TB and the overall prevalence of IR ranged between $10.7 \%$ and $27.2 \%$ (45). Likewise, in 2019 the global averages of IR without concurrent rifampicin resistance during the period 2008 to 2018 was reported to be at $7.2 \%$ and $11.6 \%$ in new TB cases and previously treated TB cases respectively (46). Therefore, detection of IR cases is equally importance because if IR cases are left undetected, they may as well impede progress being made in the fight against $\mathrm{TB}$ as new patients will be managed as if they have pan-susceptible TB. Equally, this may lead to an increased risk of treatment failure or relapse and a greater chance of acquiring further resistance.

Our study has shown that majority of DR TB patients confirmed at the reference laboratory were males, even though there was no statistical difference $(\mathrm{p}=0.452)$. Studies done in Zambia $(47)$ and elsewhere $(48,49)$ found a high prevalence of MDR TB in males than in females. This was postulated to be due to the fact that males are mostly exposed to harsh environments for a prolonged period of time (e.g., mining, prisons) which may pose a risk of having MDR TB. Equally, males tend to delay in seeking health care on account of fear of losing their jobs and income and because of alcohol dependency. 
Furthermore, TB reports from WHO and other Metadata analysis have shown the link between drug resistance and HIV AIDS $(1,39,50)$. People Living with HIV (PLHIV), especially those with lower than $200 / \mathrm{cm}^{3} \mathrm{CD} 4$ count, have a 19 (15-22) -fold risk of developing active TB compared with those who are HIV negative. According to the TB Report, 8.6\% (7.4\%-10\%) of 10 million incident cases with active TB were also co-infected with HIV in 2018 (5). Immune suppression increases the risk of reactivation of latent $\mathrm{TB}$ infection and rapid progression to active TB disease. Several biological mechanisms have been suggested linking drug resistant $\mathrm{TB}$ to $\mathrm{HIV}$ infection. These include rapid disease progression in immune compromised patients due to harboring of drug resistant strains as opposed to immune-competent individuals, drug malabsorption of anti-TB drugs, such as Rifampicin and Ethambutol, leading to drug resistance and treatment failure (3). In our study HIV co-infection was not associated with MDR TB ( $\mathrm{p}=0.396)$. Similarly, studies in Mali, Malaysia and Thailand $(41,51,52)$ found similar findings. Human Immuno-deficiency Virus testing is an essential component in the management of all presumptive drug resistance TB (DR-TB) patients. Voluntary HIV testing and counseling is recommended for all presumptive DR-TB patients, especially in high burden countries for TB and HIV coinfection. Testing for HIV should be performed simultaneously and preferably at the same center to prevent loss to follow up (4).

Lastly, our study has also reviewed that Copperbelt province has the highest proportion of MDR TB cases compared to the other two provinces. However, there was no statistical difference $(\mathrm{p}=0.093)$. According to the Zambia National TB prevalence survey report, Copperbelt Province recorded the highest prevalence of bacteriologically confirmed TB of 1, 211 per 100 000 people in the general population (47). Copperbelt Province holds the majority of copper mines in the Northern region of Zambia and the mining sector has been identified as one of the hotspots for TB transmission including drug resistant TB. Mine workers are at higher risk of contracting TB due to working conditions and prolonged exposure to silica dust (leading to development of silicosis) and high HIV prevalence in the communities $(53,54)$. Likewise, circular movement of mineworkers across provinces adversely affect adherence to TB treatment, and contribute to the incidence of drug resistant strains such as multidrug resistant (MDR) and extensively drug-resistant (XDR) TB in the province.

\section{Limitations}

The small sample size in this study may limit the generalization of the findings. This also contributed to most of the factors being not statistically significant. The review was based on the routine laboratory data generated at the regional TB reference laboratory hence other clinical factors could not be evaluated.

\section{REFERENCES}

1. WHO. WHO | Global Tuberculosis Report 2020. World Health Organization (2020). doi: 1037//0033-2909.I26.1.78

\section{CONCLUSION}

This study has shown a high proportion of MDR TB cases among drug Resistant cases in the Northern region of the country. Copperbelt province had the highest number of MDR TB Cases while North-Western province had the lowest. Over the four years under review, the proportion of Rifampicin Resistance TB cases was gradually increasing. Previous treatment and failure to treatment were strongly associated with having MDR-TB.

\section{RECOMMENDATION}

Based on the study findings, it is recommended that strengthening of reference laboratory facilities in the monitoring of MDR, pre-XDR and XDR-TB cases should be prioritized. This should include the in cooperation of phenotypic secondline DSTs and Next Generation sequencing in the diagnosis and clinical management of all MDR/RR cases. In addition, reviewing the MDR-TB situation in the whole country over a period of years in order to access progress being made in the control of MDR TB is recommended. Further, close monitoring of the treatment outcome of the cohort of MDR/ $\mathrm{RR} T B$ cases be prioritized as well as the implementation of electronic data capturing of patient clinical information and results.

\section{AUTHOR CONTRIBUTIONS}

NM contributed to the conception, reviewing literature and drafting of the manuscript. MM, MZ, MT, and RH reviewed literature and the manuscript. SM conceptualized the review, edited and supervised writing of the manuscript. All authors contributed to the article and approved the submitted version.

\section{FUNDING}

The study was funded by the African Centre of Excellence for Infectious Diseases of Humans and Animals (ACEIDHA) hosted by School of Veterinary Medicine, University of Zambia, Grant no. P151847.

\section{ACKNOWLEDGMENTS}

The authors wish to thank Tropical Diseases Research Centre for granting access to the data and the TB Regional Reference Laboratory, TDRC staff for their support during data collection. Additionally, the Africa Centre of Excellence for Infectious Diseases of Humans and Animals (ACEIDHA) for funding the research.

2. Katale BZ, Mbelele PM, Lema NA, Campino S, Mshana SE, Rweyemamu MM, et al. Whole Genome Sequencing of Mycobacterium Tuberculosis Isolates and Clinical Outcomes of Patients Treated for Multidrug-Resistant Tuberculosis in Tanzania. BMC Genomics (2020) 21(1):1-15. doi: 10.1186/s12864-020-6577-1 
3. Singh A, Prasad R, Balasubramanian V, Gupta N. Drug-Resistant Tuberculosis and Hiv Infection: Current Perspectives. HIV/AIDS - Res Palliative Care (2020) 12:9-31. doi: 10.2147/HIV.S193059

4. WHO. Companion Handbook to the WHO Guidelines for the Programmatic Management of Drug-Resistsnt Tuberculosis. Geneva: World Health Organization (2014). Available at https://apps.who.int/iris/bitstream/handle/10665/130918/ 9789241548809 Accessed 13 August 2021). WHO/CDS/TB/2019.

5. WHO. WHO Treatment Guidelines for Drug-Resistant Tuberculosi Update, October 2016 Revision (2016). World Health Organization. Available at: https://apps.who.int/iris/bitstream/handle/10665/250125/9789241549639eng.pdf (Accessed 13 August 2021). WHO/HTM/TB/2016.04.

6. Olupot-Olupot P, Namuyodi D, Obbo JSO, Meadway J. Multidrug Resistant Tuberculosis (MDR-TB) in Emerging Economies in Sub-Saharan Africa: Clinicians' Public Health Concerns. J Public Health Emergency (2017) 1:433. doi: $10.21037 /$ jphe.2017.03.01

7. Musa BM, Adamu AL, Galadanci NA, Zubayr B, Odoh CN, Aliyu MH. Trends in Prevalence of Multi Drug Resistant Tuberculosis in Sub-Saharan Africa: A Systematic Review and Meta-Analysis. PloS One (2017) 12(9):1-155. doi: 10.1371/journal.pone. 0185105

8. Ismail N, Ismail F, Omar SV, Blows L, Gardee Y, Koornhof H, et al. Drug Resistant Tuberculosis in Africa: Current Status, Gaps and Opportunities. Afr J Lab Med (2018) 7(2):1-125. doi: 10.4102/ajlm.v7i2.781

9. Migliori GB, Dheda K, Centis R, Mwaba P, Bates M, O’Grady J, et al. Review of Multidrug-Resistant and Extensively Drug-Resistant TB: Global Perspectives With a Focus on Sub-Saharan Africa. Trop Med Int Health (2010) 15(9):1052-665. doi: 10.1111/j.1365-3156.2010.02581.x

10. Lukoye D, Ssengooba W, Musisi K, Kasule GW, Cobelens FGJ, Joloba M, et al. Variation and Risk Factors of Drug Resistant Tuberculosis in Sub-Saharan Africa: A Systematic Review and Meta-Analysis. BMC Public Health (2015) 15 (1):1-13. doi: 10.1186/s12889-015-1614-8

11. Central Statistics Office. Lusaka, Zambia: Zambia Ceensus of Population and Housing (2010).

12. Kapata N, Mbulo G, Cobelens F, De Haas P, Schaap AB, Mwamba P, et al. The Second Zambian National Tuberculosis Drug Resistance Survey - A Comparison of Conventional and Molecular Methods. Tropical Med International Health (2015) 20: (11):1492-500. doi: 10.1111/tmi.12581

13. Mulenga C, Chonde A, Bwalya IC, Kapata N, Kakungu-Simpungwe M, Docx $S$, et al. Low Occurrence of Tuberculosis Drug Resistance Among Pulmonary Tuberculosis Patients From an Urban Setting, With a Long-Running DOTS Program in Zambia. Tuberculosis Res Treat (2010) 2010:1-6. doi: 10.1155/ 2010/938178

14. Kapata N, Chanda-Kapata P, Bates M, Mwaba P, Cobelens F, Grobusch MP, et al. Multidrug-Resistant TB in Zambia: Review of National Data From 2000 to 2011. Trop Med Int Health (2013) 18(11):1386-915. doi: 10.1111/tmi.12183

15. Masenga SK, Mubila H, Hamooya BM. Rifampicin Resistance in Mycobacterium Tuberculosis Patients Using GeneXpert at Livingstone Central Hospital for the Year 2015: A Cross Sectional Explorative Study. BMC Infect Dis (2017) 17(1):1-45. doi: 10.1186/s12879-017-2750-9

16. Habeenzu C, Mitarai S, Lubasi D, Mudenda V, Kantenga T, Mwansa J, et al. Tuberculosis and Multidrug Resistance in Zambian Prison-2001. Int J Tuberculosis Lung Dis (2007) 11(11):1216-205.

17. Dookie N, Rambaran S, Padayatchi N, Mahomed S, Naidoo K. Evolution of Drug Resistance in Mycobacterium Tuberculosis: A Review on the Molecular Determinants of Resistance and Implications for Personalized Care. J Antimicrobial Chemother (2018) 73(5):1138-515. doi: 10.1093/jac/dkx506

18. Meacci F, Orru G, Iona E, Giannoni F, Piersimoni C, Pozzi G, et al. Drug Resistance Evolution of a Mycobacterium Tuberculosis Strain From a Noncompliant Patient. J Clin Microbiol (2005) 43(7):3114-20. doi: 10.1128/ JCM.43.7.3114

19. Cohen KA, Abeel T, McGuire AM, Desjardins CA., Munsamy V, Shea TP, et al. Evolution of Extensively Drug-Resistant Tuberculosis Over Four Decades: Whole Genome Sequencing and Dating Analysis of Mycobacterium Tuberculosis Isolates From KwaZulu-Natal. PloS Med (2015) 12(9):1-22. doi: 10.1371/journal.pmed.1001880

20. Manson AL, Cohen KA, Abeel T, Desjardins CA, Armstrong DT, Barry CE, et al. Genomic Analysis of Globally Diverse Mycobacterium Tuberculosis Strains Provides Insights Into the Emergence and Spread of Multidrug Resistance. Nat Genet (2017) 49(3):395-402. doi: 10.1038/ng.3767
21. Hameed HM, Islam M, Chhotaray C, Wang C, Liu Y, Tan Y, et al. Molecular Targets Related Drug Resistance Mechanisms in MDR-, XDR-, and TDRMycobacterium Tuberculosis Strains. Front Cell Infect Microbiol (2018) 8:114 (APR). doi: 10.3389/fcimb.2018.00114

22. Aye KS, Nakajima C, Yamaguchi T, Win MM, Shwe MM, Win AA, et al. Genotypic Characterization of Multi-Drug-Resistant Mycobacterium Tuberculosis Isolates in Myanmar. J Infect Chemother (2016) 22(3):174795. doi: 10.1016/j.jiac.2015.12.009

23. Rozwarski DA, Grant GA, Barton DHR, Jacobs WR, Sacchettini JC. Modification of the NADH of the Isoniazid Target (InhA) From Mycobacterium Tuberculosis. Science (1998) 279(5347):98-102. doi: 10.1126/science.279.5347.98

24. Vilchèze $\mathrm{C}$, Wang F, Arai M, Hazbón MH, Colangeli R, Kremer L, et al. Transfer of a Point Mutation in Mycobacterium Tuberculosis InhA Resolves the Target of Isoniazid. Nat Med (2006) 12(9):1027-295. doi: 10.1038/nm1466

25. Zhang Y, Yew WW. Mechanism of Drug Resistance in Mycobacterium Tuberculosis. Int J Tuberc Lung Dis (2015) 19(11):1276-89. doi: 10.5588/ ijtld.15.0389

26. Muller B, Streicher ME, Hoek PGK, Tait M, Trollip A, Bosman EM, et al. InhA Promoter Mutations: A Gateway to Extensively Drug-Resistant Tuberculosis in South Africa. Int J Tuberc Lung Dis (2011) 15(3):344-51.

27. Ramaswamy SV, Reich R, Dou SJ, Jasperse L, Pan X, Wanger A, et al. Single Nucleotide Polymorphisms in Genes Associated With Isoniazid Resistance in Mycobacterium Tuberculosis. Antimicrobial Agents Chemother (2003) 47 (4):1241-505. doi: 10.1128/AAC.47.4.1241-1250.2003

28. Heep M, Rieger U, Beck D, Lehn N. Mutations in the Beginning of the RpoB Gene Can Induce Resistance to Rifamycins in Both Helicobacter Priori and Mycobacterium Tuberculosis. Antimicrobial Agents Chemother (2000) 44 (4):1075-775. doi: 10.1128/AAC.44.4.1075-1077.2000

29. Kidenya BR, Mshana SE, Fitzgerald DW, Ocheretina O. Genotypic Drug Resistance Using Whole-Genome Sequencing of Mycobacterium Tuberculosis Clinical Isolates From North-Western Tanzania. Tuberculosis (2018) 109(August 2017):97-101. doi: 10.1016/j.tube.2018.02.004

30. Saravanan M, Niguse S, Abdulkader M, Tsegay E, Hailekiros H, Gebrekidan A, et al. Review on Emergence of Drug-Resistant Tuberculosis (MDR \& XDRTB) and Its Molecular Diagnosis in Ethiopia. Microbial Pathogene (2018) 117 (February):237-42. doi: 10.1016/j.micpath.2018.02.047

31. WHO. WHO Consolidated Guidelines in Drug Resistant Tuberculosis Treatment (2019). Geneva: World Health Organization. Available at: https://apps.who.int/iris/ handle/10665/311389 (Accessed 13 August 2021). WHO/CDS/TB/2019.

32. Chisompola NK, Streicher EM, Miriam C, Muchemwa K, Warren RM, Sampson SL. Molecular Epidemiology of Drug Resistant Mycobacterium Tuberculosis in Africa: A Systematic Review. BMC Infectious Diseases (2020) 5:1-16. doi: 10.1186/s12879-020-05031-5

33. Program, Leprosy, and Clinical Care Specialist. Strengthening the GeneXpert Network: Bringing Rapid TB Testing to All in Zambia. Lusaka, FHI 360 (2018). p. 2018-20.

34. Guidelines for the Programmatic Management of Drug Resistant Tuberculosis in Zambia. (2017).

35. $\mathrm{R}$ Core Team. R: A Language and Environment for Statistical Computing. Vienna, Austria: R Foundation for Statistical Computing (2016). Available at: http://www.R-project.org/.

36. Uzoewulu NG, Ibeh IN, Lawson L, Goyal M, Umenyonu N, Ofiaeli RO, et al. Drug Resistant Mycobacterium Tuberculosis in Tertiary Hospital South East, Nigeria. J Med Microb Diagn (2014) 32. doi: 10.4172/2161-0703.1000141

37. Lv XT, Lu XW, Shi XY, Zhou L. Prevalence and Risk Factors of Multi-Drug Resistant Tuberculosis in Dalian, China. J Int Med Ress (2017) 45(6):1779-86. doi: $10.1177 / 0300060516687429$

38. Diriba G, Kabede A, Tola HH, Alemu A, Tadesse M, Tesfaye E, et al. Surveillance of Drug Resistance Tuberculosis Based on Reference Laboratory Data in Ethiopia. Infectious Diseases Poverty (2019) 8:54. doi: 10.1186/S40249-019-0554-4

39. Faustini A, Hall AJ, Perucci CA. Risk Factors for Multidrug Resistant Tuberculosis in Europe: A Systematic Review. Thorax (2006) 61(2):158-63. doi: 10.1136/thx.2005.045963

40. Daniel O, Osman E. Prevalence and Risk Factors Associated With Drug Resistant TB in South West, Nigeria. Asian Pac J Trop Med (2011) 4(2):148515. doi: 10.1016/S1995-7645(11)60057-6 
41. Elmi OS, Hasan H, Abdullah S, Jeab MZM, Alwi ZB, Naing NN. MultidrugResistant Tuberculosis and Risk Factors Associated With Its Development: A Retrospective Study. J Infect Develop Countries (2015) 9(10):1076-855. doi: 10.3855 /jidc.6162

42. Shibabaw A, Gelaw B, Gebreyes W, Robinson R, Wang SH, Tessema B. The Burden of Pre-Extensively and Extensively Drug-Resistant Tuberculosis Among MDR-TB Patients in the Amhara Region, Ethiopia. PloS One (2020) 15(2):1-135. doi: 10.1371/journal.pone.0229040

43. Daniel OJ, Osman E, Oladimeji O, Dairo OG. Pre-Extensive Drug Resistant Tuberculosis (Pre-XDR-TB) Among MDR-TB Patents in Nigeria. Global Adv Res J Microbiol (2013) 2(2):1-4.

44. Mlambo CK, Warren RM, Poswa X, Victor TC, Duse AG, Marais E. Genotypic Diversity of Extensively Drug-Resistant Tuberculosis (XDR-TB) in South Africa. Int J Tuberculosis Lung Dis (2008) 12(1):99-1045.

45. Dean AS, Zignol M, Cabibbe AM, Falzon D, Glaziou P, Cirillo DM, et al. Prevalence and Genetic Profiles of Isoniazid Resistance in Tuberculosis Patients: A Multicountry Analysis of Cross-Sectional Data. PloS Med (2020) 17(1):e1003008. doi: 10.1371/journal.pmed.1003008

46. Sulis G, Pai M. Isoniazid-Resistant Tuberculosis: A Problem We Can No Longer Ignore. PloS Med (2020) 17(1):e10030235. doi: 10.1371/journal.pmed.1003023

47. National Tuberculosis Prevalence Survey. 2013-2014 Technical Report. Zambia: Ministry of Health (2014).

48. Ibrahim E, Baess AI, Al Messery MA. Pattern of Prevalence, Risk Factors and Treatment Outcomes Among Egyptian Patients With Multidrug Resistant Tuberculosis. Egyptian J Chest Dis Tuberculosis (2017) 66(3):405-115. doi: 10.1016/j.ejcdt.2016.11.002

49. Emam SA, Kasem EM, Sedhom AE. Characteristics of Multidrug Resistant Tuberculosis in Minia, Egypt. Medico-Legal Update (2020) 20(1):446-525. doi: 10.37506/v20/i1/2020/mlu/194656

50. Pradipta IS, Forsman LD, Bruchfeld J, Hak E, Alffenaar JW. Risk Factors of Multidrug-Resistant Tuberculosis: A Global Systematic Review and MetaAnalysis. J Infect (2018) 77(6):469-785. doi: 10.1016/j.jinf.2018.10.004
51. Baya B, Achenbach CJ, Kone B, Toloba Y, Dabitao DK, Diarra B, et al. Clinical Risk Factors Associated With Multidrug-Resistant Tuberculosis (MDR-TB) in Mali. Int J Infect Dis (2019) 81:149-55. doi: 10.1016/j.ijid.2019.02.004

52. Chuchottaworn C, Thanachartwet V, Sangsayunh P, Than TZM, Sahassananda D, Surabotsophon M, et al. Risk Factors for MultidrugResistant Tuberculosis Among Patients With Pulmonary Tuberculosis at the Central Chest Institute of Thailand. PloS One (2015) 10(10):1-175. doi: 10.1371/journal.pone.0139986

53. Chanda-Kapata P, Osei-Afriyie D, Mwansa C, Kapata N. Tuberculosis in the Mines of Zambia: A Case for Intervention. Asian Pac J Trop Biomed (2016) 6 (9):803-75. doi: 10.1016/j.apjtb.2016.06.015

54. Ngosa K, Naidoo RN. The Risk of Pulmonary Tuberculosis in Underground Copper Miners in Zambia Exposed to Respirable Silica: A Cross-Sectional Study. BMC Public Health (2016) 16(1):1-75. doi: 10.1186/s12889-016-3547-2

Conflict of Interest: The authors declare that the research was conducted in the absence of any commercial or financial relationships that could be construed as a potential conflict of interest.

Publisher's Note: All claims expressed in this article are solely those of the authors and do not necessarily represent those of their affiliated organizations, or those of the publisher, the editors and the reviewers. Any product that may be evaluated in this article, or claim that may be made by its manufacturer, is not guaranteed or endorsed by the publisher.

Copyright (c) 2021 Monde, Zulu, Tembo, Handema, Munyeme and Malama. This is an open-access article distributed under the terms of the Creative Commons Attribution License (CC BY). The use, distribution or reproduction in other forums is permitted, provided the original author(s) and the copyright owner(s) are credited and that the original publication in this journal is cited, in accordance with accepted academic practice. No use, distribution or reproduction is permitted which does not comply with these terms. 